RESEARCH ARTICLE

\title{
Progressive First Degree AV Block Indicates the Need for Permanent Pacing
}

\author{
Han B Xiao*, Waleed Arshad, Arvinder Kurbaan and Timothy J Bowker \\ Department of Cardiology, Homerton University Hospital, UK
}

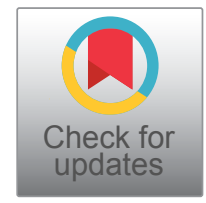

*Corresponding author: Han B Xiao, MBBS, PhD, FESC, FRCP, Consultant Cardiologist, Department of Cardiology, Homerton University Hospital, Homerton Row, London E9 6SR, UK, Tel: 0208-510-5555, Fax: 020-8510-7687

\begin{abstract}
Aims: First-degree AV block, though considered benign, has been reported to be associated with an increased risk for atrial fibrillation, permanent pacing, heart failure and all-cause mortality. It is possible that the extent of PR prolongation indicates the severity of adverse outcome. This study investigated whether the length of PR and its change with heart rate can predict the need for permanent pacing.

Method: We retrospectively studied 21 patients with a PR over $270 \mathrm{~ms}$. Nine patients underwent permanent pacing for symptomatic bradycardia or complete AV block and 12 did not. Two or more PR intervals and corresponding heart rates were measured. The unit change in PR to heart rate was calculated and defined as PR Index. We compared the two groups by Student $\mathrm{T}$ test or Fisher Chi Square test.

Result: Age, sex distribution and baseline heart rate were similar in the two groups. The baseline mean PR interval was $70 \mathrm{~ms}$ greater (though did not reach statistical significance), a PR interval over $350 \mathrm{~ms}$ was more common (7/9 vs. 3/12), the mean corrected PR interval by heart rate was significantly longer and the PR index was significantly higher in patients who were paced than in those who were not (all $p<0.05)$.

Conclusion: A very long PR interval may lead to AV dissociation requiring permanent pacing. A PR interval over $350 \mathrm{~ms}$ and an exaggerated prolongation of PR per given decrease in heart rate may indicate a high risk of $A V$ dissociation, hence the need for permanent pacing
\end{abstract}

\section{Keywords}

PR interval, PR Index, First degree atrioventricular block, Pacing

\section{Introduction}

In the history of electrocardiography, time intervals have aroused considerable interest. The QT interval and QRS duration have been extensively investigated $[1,2]$, and the PR interval [2-4], less so. There is a general consensus that the PR intervals up to 200 $\mathrm{ms}$ are normal. But the criterion above which first degree atrioventricular (AV) block is defined ranges from 200 to $220 \mathrm{~ms}$ [5-7]. First degree AV block is common and its prevalence increases with age [8-10]. Although first degree AV block is generally considered benign $[10,11]$, some longitudinal studies suggest that a prolonged PR interval is associated with an increased frequency of atrial fibrillation, pacemaker implantation, heart failure and all-cause mortality $[9,12,13]$. It is not clear if there is a direct graded relationship between the extent of $P R$ prolongation and the severity of adverse outcome, namely the longer the PR interval the poorer the prognosis. A long PR interval $\geq 300 \mathrm{~ms}$ has a prevalence of as low as $<1 / 10,000$ [10] but can cause symptoms warranting permanent pacing [13]. In any given individual, there is a physiological inverse relationship between heart rate and PR interval duration [14-16] and we have recently proposed that the gradient with which the $P R$ interval changes per unit change in heart rate may be of assistance in the prediction of outcome and thus possibly of the need for subsequent cardiac pacing [14]. In order to explore further this possibility in patients with a very long PR interval, we have analysed all patients with a PR interval of over 270 ms seen in our department over a 2.5 year period.

\section{Method}

From February 2015 to August 2017, 25 patients with a PR interval over $270 \mathrm{~ms}$ were seen in our

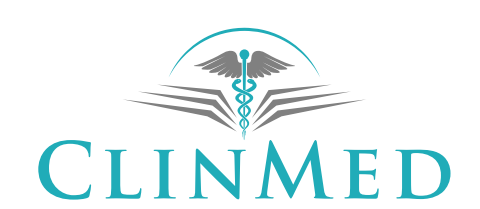

INTERNATIONAL LIBRARY
Citation: Xiao HB, Arshad W, Kurbaan A, Bowker TJ (2019) Progressive First Degree AV Block Indicates the Need for Permanent Pacing. Int J Clin Cardiol 6:162. doi.org/10.23937/2378-2951/1410162 Accepted: November 25, 2019; Published: November 27, 2019

Copyright: (c) 2019 Xiao HB, et al. This is an open-access article distributed under the terms of the Creative Commons Attribution License, which permits unrestricted use, distribution, and reproduction in any medium, provided the original author and source are credited. 
Table 1: Comparison between patients who were paced and those who were not.

\begin{tabular}{|c|c|c|c|}
\hline & Patients paced $(n=9)$ & Patients not paced $(n=12)$ & P Value \\
\hline Age (years) & $75 \pm 15$ & $69 \pm 22$ & NS \\
\hline Male/Female & 1-Aug & 2-Sep & NS \\
\hline Baseline heart rate (HR, bpm) & $58 \pm 20$ & $60 \pm 10$ & NS \\
\hline Baseline PR (PR, ms) & $405 \pm 102$ & $337 \pm 40$ & NS \\
\hline PR > $350 \mathrm{~ms}$ & $7(78 \%)$ & $3(25 \%)$ & $<0.05$ \\
\hline Baseline PRc1 (ms) & $395 \pm 62$ & $335 \pm 45$ & $<0.05$ \\
\hline Baseline PRc2 (ms) & $385 \pm 60$ & $340 \pm 45$ & NS \\
\hline Peak heart rate (HRmax, bpm) & $82 \pm 20$ & $117 \pm 27$ & $<0.01$ \\
\hline PR at HRmax (PRmin, ms) & $320 \pm 85$ & $200 \pm 40$ & $<0.05$ \\
\hline PRc1 at HRmax ( $\mathrm{ms}$ ) & $370 \pm 85$ & $275 \pm 40$ & $<0.05$ \\
\hline PRc2 at HRmax (ms) & $336 \pm 132$ & $218 \pm 35$ & $<0.05$ \\
\hline PR index (ms/10 bpm) & $40 \pm 12$ & $28 \pm 12$ & $<0.05$ \\
\hline
\end{tabular}

PRc1: PR interval corrected by heart rate alone; PRc2: PR interval corrected by both age and heart rate.

department. We retrospectively reviewed all these cases without knowledge of the clinical management plan decided by the patients respective consultants. All patients had a resting 12 lead ECG, twelve patients underwent a 24 hour ECG, two patients a 48 hour ECG, four an exercise tolerance ECG and three a dobutamine stress echocardiogram. Four patients were excluded from this study, one had a PPM and one ICD with pacing implanted prior to this project and 2 patients had only one resting ECG available. Thus, 21 patients who had a 12 lead ECG and at least one other ECG of various forms allowing measurement of two or more PR intervals and corresponding heart rates were studied, and their clinical and ECG data were analysed.

Nine of the 21 patients underwent permanent pacing for symptomatic bradycardia, 5 developed complete AV block, 3 had syncopal attacks, one had a symptomatic long sinus pause of 4.2 seconds. Therefore the 9 patients who had a pacemaker and the remaining 12 patients in this cohort were naturally formed two groups.

We measured the baseline PR interval (PRmax) and heart rate (HRmin) on the first, invariably resting ECG. We measured a second PR interval (PRmin) at the highest heart rate (HRmax) on the subsequently ECGs available.

We then obtained the following indices.

Corrected PR intervals (PRc1 and PRc2) by two methods on both resting ECG and the ECG with the highest heart rate recorded:

PRc1: the PR interval was corrected by heart rate (RR) alone in the way the QT interval is corrected [17],

$\mathrm{PRc1}=\mathrm{PR} / \mathrm{V}(\mathrm{RR})$.

PRc2: the PR interval was corrected by both age and heart rate according to Soliman, et al. [4].
For age $>60$ years: $\mathrm{PRc} 2=\mathrm{PR}+0.42(\mathrm{HR}-70)$

For age $<60$ years: $P R c 2=P R+0.26(H R-70)$

We defined the PR index as the change in PR interval for every 10 beats per minute change in heart rate (ms/10 bpm): [(PR max - PR min) / (HR max - HR min)] $\times 10$.

We compared patients who have been paced with those who have not been paced by Student $T$ test or Fisher Chi Square test. A P value of $<0.05$ was considered as statistically significant.

\section{Result}

The patients' ages ranged from 43 to 90 -years. The majority $(18 / 21)$ were male (Table 1$)$. In order to investigate if any parameters are associated with complete AV block or with significant bradycardia, and thus the need of pacing, we compared patients who have been paced with those who have not been paced. There was no difference in age, sex distribution and baseline heart rate. The baseline mean PR interval was $70 \mathrm{~ms}$ greater in patients who were paced than in those who were not, but the difference did not reach statistical significance. However, the proportion of patients with a PR interval over $350 \mathrm{~ms}$ at baseline was significantly higher in patients who were paced than in those who were not $(7 / 9$ vs. $3 / 12, P<0.05)$. In the whole patient population, of the 10 patients with a PR interval over $350 \mathrm{~ms}, 7$ of them underwent subsequent pacemaker implantation and 3 did not, while of the 11 patients with a PR interval $\leq 350 \mathrm{~ms}$, only 2 were paced and the remaining 9 patients did not require pacing. This indicates a PR interval over $350 \mathrm{~ms}$ is associated with the subsequent high degree AV block, hence the need for pacing.

At baseline, when corrected by heart rate alone the mean corrected PR interval (PRc1 max) was significantly longer in patients who were paced $(P$ $<0.05$ ), but when corrected by both age and heart 
rate, the baseline mean corrected PR interval (PRc2 max) was not significantly longer in pace patients. However, at the highest heart rates, the PR interval corrected by heart rate alone (PRc2 $\mathrm{min}$ ) or by both age and heart rate (PRc2 $\mathrm{min}$ ) was longer in patients who were paced than in those who were not paced (all $\mathrm{P}<0.05)$. Importantly, the PR index was significantly higher in those who were paced than in those who were not $(p<0.05)$ (Table 1$)$. This indicated a greater $\mathrm{PR}$ prolongation per unit reduction in heart rate is associated a greater need for pacing.

\section{Discussion}

The PR interval shortens with rising heart rate in response to exercise or to other forms of stress $[15,16,18]$. This relationship pertains in all subjects both on resting ECGs and on 24 hour ECGs, possibly representing physiological circadian variability. The nature of the relationship of the PR interval to heart rate could provide prognostic information, particularly in the presence of pre-existing PR prolongation, itself already associated with an adverse prognosis $[9,12,13,19]$. However, it remains unclear to what extent an exceptionally long PR interval is a prelude to advanced AV block requiring permanent pacing [14]. The current observational clinical survey explores this.

The prevalence in the general population of $P R$ intervals $>300 \mathrm{~ms}$ is estimated to be as low as $<1 / 10,000$ [10]. The prevalence of PR intervals over 350 ms would be even lower, though such data are not available. We investigated all patients with a PR interval > $270 \mathrm{~ms}$ seen in our department over a 2.5 year period. Some of these patients were paced according to their clinical features and/or in response to advice from tertiary centre specialist electrophysiologists who were not participating in the current clinical survey.

A baseline PR interval over 350 ms, higher mean corrected PR interval, and higher PR index were each associated the more likelihood of subsequent permanent endocardial pacing.

Previous studies have shown an inverse relation between heart rate and the length of PR interval in normal subjects and in patients $[15,16,18]$, even in those with a very long PR interval [14]. The relationship between $P R$ interval and heart rate during stress can be influenced by sympathetic tone [18], but worsening AV node dysfunction is the likely mechanism by which a long PR interval progresses to AV dissociation, particularly if there is no evidence of abnormal sympathetic drive. We feel that the patients in our current study demonstrate varying degrees of AV nodal dysfunction. One of the manifestations of such AV nodal dysfunction may be the progressive loss of the normal physiological dynamic relationship between PR interval and heart rate.
Thus, while it is logical to think that a long PR interval which lengthens progressively over time will ultimately lead to complete AV dissociation and thus to the need of pacing, it could be that the PR to HR inter-relationship, defined as "PR index" in this study, also indicates the need of pacing. The natural inverse relationship between heart rate and $P R$ interval usually operates within a normal physiological range, thereby delivering a physiological heart rate response to external and internal stimuli. With the passage of time, in some patients disturbance of this physiological relationship may occur, such that for a given reduction in heart rate there is an exaggerated prolongation in $\mathrm{PR}$ interval. In our patients, the increase in PR interval per unit reduction in heart rate (the PR index) was greater in those who were subsequently paced than in those who were not. Careful measurement of the quantitative value of the natural inverse relationship between PR interval and heart rate, or of its derivatives (e.g. the PR index) may help identify a sub-group of subjects at higher risk of the subsequent development of AV dissociation, and who thus might benefit from a lowering of the threshold for permanent endocardial pacing.

Other groups have similarly concluded that a long PR interval leads to complete AV dissociation $[9,13]$. In addition, our previous finding that in patients with a very long PR interval, the inverse relationship between $P R$ interval and heart rate is steeper than that in control subjects indicates that the slope of $\mathrm{PR} /$ heart rate relationship on stress testing could help determine the need for pacing, particularly in those who have not yet developed complete AV block [14].

The PR interval to heart rate relationship, in terms of regression coefficient, differs between the normal population (-0.5 to -0.66$)[15,18]$ and patients with a very long PR interval (-2.26) [14]. In the current study, only some of the patients had a stress test as part of the clinical observations, the regression coefficient was not performed, instead we calculated the PR index. The association of a higher PR index with subsequent need for pacing suggests that the value of the slope of the PR/heart rate relationship can be used to help determine the need for pacing, maybe even before the patient develops any symptoms.

One may ask if the approach of the PR to heart rate relationship can be employed beyond patients with very long PR intervals, such as in patients with only mild $1^{\text {st }}$ degree AV block, in the investigation of syncope or of pre-syncope, or even in the assessment of the safety of introducing beta-blockade (or other AV node inhibitory agents) in specific sub-groups. The current study is unable to address such questions quantitatively but this line of observation may well be fruitful.

The major limitations of this study are of two fold, 
the small sample size and the lack of a stress test in some patients. It is understandable that the sample size is small due to the extremely low prevalence of an exceptionally long PR interval and to the study having been carried out in a single institute. This study may encourage further observations by specialist centres to clarify the indication of permanent pacing in such patients. We could not carry out stress tests in all our patients for the purpose of research as we only planned to carry out a clinical survey without influencing the actual clinical management of the patients who were cared for by different cardiologists.

In conclusion, in our study population as a whole, a very long PR interval (greater than $350 \mathrm{~ms}$ ) was associated with subsequent need for permanent pacing. Observing heart rate changes within individual subjects, the increase in the PR interval per unit reduction in heart rate (the PR index) was greater in those who were subsequently paced than in those who were not. We believe that further quantitative analysis of the dynamic relationship between PR interval and heart rate on stress testing, even at an early stage, may help distinguish patients who are in need of a permanent pacemaker from those who are not.

\section{Declaration of Interest}

None.

\section{References}

1. Packard JM, Graettinger JS, Graybiel A (1954) Analysis of the electrocardiograms obtained from 1000 young healthy aviators: Ten year follow-up. Circulation 10: 384-400.

2. Malik M, Hnatkova K, Sisakova M, Schmidt G (2008) Subjectspecific heart rate dependency of electrocardiographic QT, $P Q$, and QRS intervals. J Electrocardiol 41: 491-497.

3. Mason JW, Ramseth DJ, Chanter DO, Moon TE, Goodman D, et al. (2007) Electrocardiographic reference ranges derived from 79,743 ambulatory subjects. J Electrocardiol 40: 228-234.

4. Soliman EZ, Rautaharju PM (2012) Heart rate adjustment of PR interval in middle-aged and older adults. J Electrocardiol 45: 66-69.

5. Soliman EZ, Alonso A, Misialek JR, Jain A, Watson KE, et al. (2013) Reference ranges of PR duration and P-wave indices in individuals free of cardiovascular disease: The Multi-Ethnic Study of Atherosclerosis (MESA). J Electrocardiol 46: 702-706.

6. Epstein AE, Dimarco JP, Ellenbogen KA, Estes NA, Freedman RA, et al. (2008) ACC/AHA/HRS 2008 Guidelines for device-based therapy of cardiac rhythm abnormalities. A report of the american college of cardiology/american heart association task force on practice guidelines (writing committee to revise the ACC/AHA/NASPE 2002 guideline update for implantation of cardiac pacemakers and antiarrhythmia devices): Developed in collaboration with the american association for thoracic surgery and society of thoracic surgeons. Circulation 117: e350-e408.

7. Nada A, Gintant GA, Kleiman R, Gutstein DE, Gottfridsson C, et al. (2013) The evaluation and management of drug effects on cardiac conduction (PR and QRS Intervals) in clinical development. Am Heart J 165: 489-500.

8. Hiss RG, Lamb LE (1962) Electrocardiographic findings in 122,043 individuals. Circulation 25: 947-961.

9. Cheng S, Keyes MJ, Larson MG, McCabe EL, NewtonCheh C, et al. (2009) Long-term outcomes in individuals with prolonged pr interval or first-degree atrioventricular block. JAMA 301: 2571-2577.

10. Aro AL, Anttonen O, Kerola T, Junttila MJ, Tikkanen JT, et al. (2014) Prognostic significance of prolonged PR interval in the general population. Eur Heart J 35: 123-129.

11. Erekssen J, Otterstad JE (1984) Natural course of a prolonged $\mathrm{pr}$ interval and the relation between $\mathrm{pr}$ and incidence of coronary heart disease. A 7-year follow-up study of 1832 apparently healthy men aged 40-59-years. Clin Cardiol 7: 6-13.

12. Crisel RK, Farzaneh-Far R, Na B, Whooley MA (2011) First-degree atrioventricular block is associated with heart failure and death in persons with stable coronary artery disease: Data from the heart and soul study. Eur Heart $\mathrm{J}$ 32: 1875-1880.

13. Barold SS, llercil A, Leonelli F, Herweg B (2006) First-degree atrioventricular block. Clinical manifestations, indications for pacing, pacemaker management \& consequences during cardiac resynchronization. J Interv Card Electrophysiol 17: 139-152.

14. Ramoutar A, Bowker T, Kurbaan A, Xiao HB (2015) When is a PR interval too long? Int J Cardiol 199: 42-43.

15. Carruthers SG, McCall B, Cordell BA, Wu R (1987) Relationships between heart rate and $P R$ interval during physiological and pharmacological interventions. Br J Clin Pharmacol 23: 259-265.

16. Danter WR, Carruthers SG (1990) The heart rate-PR interval relationship: A model for evaluating drug actions on SA and AV nodal function. Br J Clin Pharmacol 30: 490492.

17. Bazett HC (1920) An analysis of the time-relations of electrocardiograms. Heart 7: 353-370.

18. Lee JU, Kim KS, Kim JH, Lim HK, Lee BH, et al. (1995) PR interval behaviour during exercise stress test. Korean $\mathrm{J}$ Intern Med 10: 137-142.

19. Nikolaidou T, Ghosh JM, Clark AL (2016) Outcomes related to first-degree atrioventricular block and therapeutic implications in patients with heart failure. JACC Clin Electrophysiol 2: 181-192. 